\title{
Constructive description of monogenic functions in a finite-dimensional commutative associative algebra
}

\author{
V. S. Shpakivskyi
}

\begin{abstract}
Let $\mathbb{A}_{n}^{m}$ be an arbitrary $n$-dimensional commutative associative algebra over the field of complex numbers with $m$ idempotents. Let $e_{1}=1, e_{2}, e_{3}$ be elements of $\mathbb{A}_{n}^{m}$ which are linearly independent over the field of real numbers. We consider monogenic (i. e. continuous and differentiable in the sense of Gateaux) functions of the variable $x e_{1}+y e_{2}+z e_{3}$, where $x, y, z$ are real, and obtain a constructive description of all mentioned functions by means of holomorphic functions of complex variables. It follows from this description that monogenic functions have Gateaux derivatives of all orders.
\end{abstract}

Keywords: Commutative associative algebra; monogenic function; constructive description.

\section{Introduction.}

An effectiveness of the analytic function methods in the complex plane for researching plane potential fields inspires mathematicians to develop analogous methods for spatial fields.

Apparently, W. Hamilton (1843) made the first attempts to construct an algebra associated with the three-dimensional Laplace equation

$$
\Delta_{3} u(x, y, z):=\left(\frac{\partial^{2}}{\partial x^{2}}+\frac{\partial^{2}}{\partial y^{2}}+\frac{\partial^{2}}{\partial z^{2}}\right) u(x, y, z)=0
$$

meaning that components of hypercomplex functions satisfy the equation (1). He constructed an algebra of noncommutative quaternions over the field of real numbers $\mathbb{R}$ and made a base for developing the hypercomplex analysis.

C. Segre [1] constructed an algebra of commutative quaternions over the field $\mathbb{R}$ that can be considered as a two-dimensional commutative semi-simple algebra of bicomplex numbers over the field of complex numbers $\mathbb{C}$. F. Ringleb [2] and J. Riley [3] obtained a constructive description of analytic function of a bicomplex variable, namely, they proved that such an analytic function can be constructed with an use of two holomorphic functions of complex variables. 
Furthermore, F. Ringleb [2] considered an arbitrary finite-dimensional associative (commutative or not) semi-simple algebra over the field $\mathbb{R}$. For analytic functions which maps the mentioned algebra onto itself, he obtained a constructive description by means of real and complex analytic functions.

A relation between spatial potential fields and analytic functions given in commutative algebras was established by P. W. Ketchum [4] who shown that every analytic function $\Phi(\zeta)$ of the variable $\zeta=x e_{1}+y e_{2}+z e_{3}$ satisfies the equation (1) in the case where the elements $e_{1}, e_{2}, e_{3}$ of a commutative algebra satisfy the condition

$$
e_{1}^{2}+e_{2}^{2}+e_{3}^{2}=0
$$

because

$$
\frac{\partial^{2} \Phi}{\partial x^{2}}+\frac{\partial^{2} \Phi}{\partial y^{2}}+\frac{\partial^{2} \Phi}{\partial z^{2}} \equiv \Phi^{\prime \prime}(\zeta)\left(e_{1}^{2}+e_{2}^{2}+e_{3}^{2}\right)=0
$$

where $\Phi^{\prime \prime}:=\left(\Phi^{\prime}\right)^{\prime}$ and $\Phi^{\prime}(\zeta)$ is defined by the equality $d \Phi=\Phi^{\prime}(\zeta) d \zeta$.

We say that a commutative associative algebra $\mathbb{A}$ is harmonic (cf. [4, 5, 7]) if in $\mathbb{A}$ there exists a triad of linearly independent vectors $\left\{e_{1}, e_{2}, e_{3}\right\}$ satisfying the equality (2) with $e_{k}^{2} \neq 0$ for $k=1,2,3$. We say also that such a triad $\left\{e_{1}, e_{2}, e_{3}\right\}$ is harmonic.

P. W. Ketchum [4] considered the C. Segre algebra of quaternions [1] as an example of harmonic algebra.

I. P. Mel'nichenko [5] noticed that doubly differentiable in the sense of Gateaux functions form the largest algebra of functions $\Phi$ satisfying identically the equalities (3), where $\Phi^{\prime \prime}$ is the Gateaux second derivative of function $\Phi$. He proved that there exist exactly 3 three-dimensional harmonic algebras with unit over the field $\mathbb{C}$ (see [5, 6, 7]).

Constructive descriptions of monogenic (i. e. continuous and differentiable in the sense of Gateaux) functions taking values in the mentioned threedimensional harmonic algebras by means three corresponding holomorphic functions of the complex variable are obtained in the papers [8, 9, 10]. Such descriptions make it possible to prove the infinite differentiability in the sense of Gateaux of monogenic functions and integral theorems for these functions that 
are analogous to classical theorems of the complex analysis (see, e. g., [11, 12]).

Furthermore, constructive descriptions of monogenic functions taking values in special $n$-dimensional commutative algebras by means $n$ holomorphic functions of complex variables are obtained in the papers [13, 14].

In this paper we obtaine a constructive description of monogenic functions taking values in an arbitrary finite-dimensional commutative associative algebra with unit by means of holomorphic functions of complex variables. It follows from this description that the monogenic functions have the Gateaux derivatives of all orders.

\section{The algebra $\mathbb{A}_{n}^{m}$.}

Let $\mathbb{N}$ be the set of natural numbers. We fix the numbers $m, n \in \mathbb{N}$ such that $m \leq n$. Let $\mathbb{A}_{n}^{m}$ be an arbitrary commutative associative algebra with unit over the field of complex number $\mathbb{C}$. E. Cartan [15, p. 33] proved that there exist a basis $\left\{I_{k}\right\}_{k=1}^{n}$ in $\mathbb{A}_{n}^{m}$ satisfying the following multiplication rules:

1. $\forall r, s \in[1, m] \cap \mathbb{N}: \quad I_{r} I_{s}=\left\{\begin{array}{cc}0 & \text { if } r \neq s \\ I_{r} & \text { if } r=s\end{array}\right.$

2. $\forall r, s \in[m+1, n] \cap \mathbb{N}: \quad I_{r} I_{s}=\sum_{k=\max \{r, s\}+1}^{n} \Upsilon_{r, k}^{s} I_{k}$

3. $\forall s \in[m+1, n] \cap \mathbb{N} \quad \exists ! u_{s} \in[1, m] \cap \mathbb{N} \quad \forall r \in[1, m] \cap \mathbb{N}$ :

$$
I_{r} I_{s}=\left\{\begin{array}{l}
0 \text { if } r \neq u_{s} \\
I_{s} \text { if } r=u_{s}
\end{array}\right.
$$

Moreover, the structure constants $\Upsilon_{r, k}^{s} \in \mathbb{C}$ satisfy the associativity conditions:

(A 1). $\left(I_{r} I_{s}\right) I_{p}=I_{r}\left(I_{s} I_{p}\right) \quad \forall r, s, p \in[m+1, n] \cap \mathbb{N}$

(A 2). $\left(I_{u} I_{s}\right) I_{p}=I_{u}\left(I_{s} I_{p}\right) \quad \forall u \in[1, m] \cap \mathbb{N} \quad \forall s, p \in[m+1, n] \cap \mathbb{N}$.

Obviously, the first $m$ basic vectors $\left\{I_{u}\right\}_{u=1}^{m}$ are idempotents and form a semi-simple subalgebra of the algebra $\mathbb{A}_{n}^{m}$. The vectors $\left\{I_{r}\right\}_{r=m+1}^{n}$ form a nilpotent subalgebra of the algebra $\mathbb{A}_{n}^{m}$. The element $1=\sum_{u=1}^{m} I_{u}$ is the unit 
of $\mathbb{A}_{n}^{m}$.

In the cases where $\mathbb{A}_{n}^{m}$ has some specific properties, the following propositions are true.

Proposition 1. If there exists the unique $u_{0} \in[1, m] \cap \mathbb{N}$ such that $I_{u_{0}} I_{s}=I_{s}$ for all $s=m+1, \ldots, n$, then the associativity condition (A 2) is satisfied.

Proof. In the associativity condition (A 2), two cases are possible:

$$
\begin{array}{ll}
\text { 1) } & I_{u} \neq I_{u_{0}}, \text { then } I_{u} I_{s}=0 \quad \forall s=m+1, \ldots, n ; \\
\text { 2) } & I_{u}=I_{u_{0}}, \text { then } I_{u} I_{s}=I_{s} \quad \forall s=m+1, \ldots, n .
\end{array}
$$

In the first case, the condition (A 2) takes the form

$$
0 \cdot I_{p}=I_{u} \sum_{k=\max \{s, p\}+1}^{n} \Upsilon_{s, k}^{p} I_{k}=0
$$

because $I_{u} I_{k}=0$ for all $k=\max \{s, p\}+1, \ldots, n$.

In the second case, the condition (A 2) takes the form

$$
I_{s} I_{p}=I_{u} \sum_{k=\max \{s, p\}+1}^{n} \Upsilon_{s, k}^{p} I_{k} .
$$

It is equivalent to the equality

$$
\sum_{k=\max \{s, p\}+1}^{n} \Upsilon_{s, k}^{p} I_{k}=\sum_{k=\max \{s, p\}+1}^{n} \Upsilon_{s, k}^{p} I_{k},
$$

because $I_{u} I_{k}=I_{k}$ for all $k=\max \{s, p\}+1, \ldots, n$. The proposition is proved.

Thus, under the conditions of Proposition 1, the associativity condition (A 1) is only required. It means that the nilpotent subalgebra of $\mathbb{A}_{n}^{m}$ with the basis $\left\{I_{r}\right\}_{r=m+1}^{n}$ can be an arbitrary commutative associative nilpotent algebra of dimension $n-m$. Note that such nilpotent algebras are fully described for the dimensions 1, 2, 3, 4 in the paper [16].

Proposition 2. If all $u_{r}$ are different in the multiplication rule 3 , then $I_{s} I_{p}=0$ for all $s, p=m+1, \ldots, n$.

Proof. Let $s \in[m+1, n] \cap \mathbb{N}$. We choose $I_{u}$ such that $I_{u} I_{s}=I_{s}$. Then from the associativity condition (A 2) we obtain the identity

$$
I_{s} I_{p}=I_{u} \sum_{k=\max \{s, p\}+1}^{n} \Upsilon_{s, k}^{p} I_{k}=0,
$$


because by assumption of theorem $I_{u} I_{k}=0$ for all $k=\max \{s, p\}+1, \ldots, n$. The proposition is proved.

Thus, under the conditions of Proposition 2, the multiplication table of the nilpotent subalgebra of $\mathbb{A}_{n}^{m}$ with the basis $\left\{I_{r}\right\}_{r=m+1}^{n}$ consists only of zeros, and all associativity conditions are satisfied.

The algebra $\mathbb{A}_{n}^{m}$ contains $m$ maximal ideals

$$
\mathcal{I}_{u}:=\left\{\sum_{k=1, k \neq u}^{n} \lambda_{k} I_{k}: \lambda_{k} \in \mathbb{C}\right\}, \quad u=1,2, \ldots, m,
$$

and their intersection is the radical

$$
\mathcal{R}:=\left\{\sum_{k=m+1}^{n} \lambda_{k} I_{k}: \lambda_{k} \in \mathbb{C}\right\} .
$$

Consider $m$ linear functionals $f_{u}: \mathbb{A}_{n}^{m} \rightarrow \mathbb{C}$ satisfying the equalities

$$
f_{u}\left(I_{u}\right)=1, \quad f_{u}(\omega)=0 \quad \forall \omega \in \mathcal{I}_{u}, \quad u=1,2, \ldots, m .
$$

Inasmuch as the kernel of functional $f_{u}$ is the maximal ideal $\mathcal{I}_{u}$, this functional is also continuous and multiplicative (see [17, p. 147]).

\section{Monogenic functions.}

We consider the vectors $e_{1}=1, e_{2}, e_{3}$ in $\mathbb{A}_{n}^{m}$ which are linearly independent over the field of real numbers $\mathbb{R}$. It means that the equality

$$
\alpha_{1} e_{1}+\alpha_{2} e_{2}+\alpha_{3} e_{3}=0, \quad \alpha_{1}, \alpha_{2}, \alpha_{3} \in \mathbb{R},
$$

holds if and only if $\alpha_{1}=\alpha_{2}=\alpha_{3}=0$.

Let the vectors $e_{1}=1, e_{2}, e_{3}$ have the following decompositions with respect to the basis $\left\{I_{k}\right\}_{k=1}^{n}$ :

$$
e_{1}=\sum_{r=1}^{m} I_{r}, \quad e_{2}=\sum_{r=1}^{n} a_{r} I_{r}, \quad e_{3}=\sum_{r=1}^{n} b_{r} I_{r},
$$

where $a_{r}, b_{r} \in \mathbb{C}$.

Let $\zeta:=x e_{1}+y e_{2}+z e_{3}$, where $x, y, z \in \mathbb{R}$. It is obvious that $\xi_{u}:=f_{u}(\zeta)=$ $x+y a_{u}+z b_{u}, u=1,2, \ldots, m$. Let $E_{3}:=\left\{\zeta=x e_{1}+y e_{2}+z e_{3}: x, y, z \in \mathbb{R}\right\}$ be the linear span of vectors $e_{1}, e_{2}, e_{3}$ over the field $\mathbb{R}$. 
Let $\Omega$ be a domain in $\mathbb{R}^{3}$. Associate with $\Omega$ the domain $\Omega_{\zeta}:=\{\zeta=$ $\left.x e_{1}+y e_{2}+z e_{3}:(x, y, z) \in \Omega\right\}$ in $E_{3}$.

We say that a continuous function $\Phi: \Omega_{\zeta} \rightarrow \mathbb{A}_{n}^{m}$ is monogenic in $\Omega_{\zeta}$ if $\Phi$ is differentiable in the sense of Gateaux in every point of $\Omega_{\zeta}$, i. e. if for every $\zeta \in \Omega_{\zeta}$ there exists an element $\Phi^{\prime}(\zeta) \in \mathbb{A}_{n}^{m}$ such that

$$
\lim _{\varepsilon \rightarrow 0+0}(\Phi(\zeta+\varepsilon h)-\Phi(\zeta)) \varepsilon^{-1}=h \Phi^{\prime}(\zeta) \quad \forall h \in E_{3}
$$

$\Phi^{\prime}(\zeta)$ is the Gateaux derivative of the function $\Phi$ in the point $\zeta$.

In the scientific literature the denomination of monogenic function is used else for functions satisfying certain conditions similar to the classical Cauchy Riemann conditions (see, e. g., [18, 19]). Such functions are also called regular functions (see [20]) or hyperholomorphic functions (see, e. g., [21, 22]).

Consider the decomposition of a function $\Phi: \Omega_{\zeta} \rightarrow \mathbb{A}_{n}^{m}$ with respect to the basis $\left\{I_{k}\right\}_{k=1}^{n}$ :

$$
\Phi(\zeta)=\sum_{k=1}^{n} U_{k}(x, y, z) I_{k} .
$$

In the case where the functions $U_{k}: \Omega \rightarrow \mathbb{C}$ are $\mathbb{R}$-differentiable in $\Omega$, i. e. for every $(x, y, z) \in \Omega$,

$$
\begin{aligned}
& U_{k}(x+\Delta x, y+\Delta y, z+\Delta z)-U_{k}(x, y, z)=\frac{\partial U_{k}}{\partial x} \Delta x+\frac{\partial U_{k}}{\partial y} \Delta y+\frac{\partial U_{k}}{\partial z} \Delta z+ \\
& +o\left(\sqrt{(\Delta x)^{2}+(\Delta y)^{2}+(\Delta z)^{2}}\right), \quad(\Delta x)^{2}+(\Delta y)^{2}+(\Delta z)^{2} \rightarrow 0,
\end{aligned}
$$

the function $\Phi$ is monogenic in the domain $\Omega_{\zeta}$ if and only if the following Cauchy - Riemann conditions are satisfied in $\Omega_{\zeta}$ :

$$
\frac{\partial \Phi}{\partial y}=\frac{\partial \Phi}{\partial x} e_{2}, \quad \frac{\partial \Phi}{\partial z}=\frac{\partial \Phi}{\partial x} e_{3}
$$

Below, it will be shown that all components $U_{k}$ of the monogenic function (7) are infinitely $\mathbb{R}$-differentiable in $\Omega$. 


\section{An expansion of the resolvent.}

Lemma 1. An expansion of the resolvent is of the form

$$
\left(t e_{1}-\zeta\right)^{-1}=\sum_{r=1}^{n} A_{r} I_{r} \quad \forall t \in \mathbb{C}: t \neq \xi_{u}, \quad u=1,2, \ldots, m,
$$

with the coefficients $A_{r}$ are determined by the following recurrence relations:

$$
\begin{gathered}
A_{u}=\frac{1}{t-\xi_{u}}, \quad u=1,2, \ldots, m, \quad A_{m+1}=\frac{T_{m+1}}{\left(t-\xi_{u_{m+1}}\right)^{2}}, \\
A_{p}=\frac{T_{p}}{\left(t-\xi_{u_{p}}\right)^{2}}+\frac{1}{t-\xi_{u_{p}}} \sum_{r=m+1}^{p-1} A_{r} B_{r, p}, \quad p=m+2, m+3, \ldots, n,
\end{gathered}
$$

where

$$
\begin{aligned}
T_{p} & :=y a_{p}+z b_{p}, \quad p=m+1, m+2, \ldots, n, \\
B_{r, p} & :=\sum_{s=m+1}^{p-1} T_{s} \Upsilon_{r, p}^{s}, \quad p=m+2, m+3, \ldots, n,
\end{aligned}
$$

and the natural numbers $u_{p}$ are defined in the rule 3 of the multiplication table of algebra $\mathbb{A}_{n}^{m}$.

Proof. Let's find $t \in \mathbb{C}$ for which there exists the element $\left(t e_{1}-\zeta\right)^{-1}$ in the algebra $\mathbb{A}_{n}^{m}$ and let's find the coefficients $A_{r}$ of decomposition of this element with respect to the basis $\left\{I_{k}\right\}_{k=1}^{n}$ :

$$
\left(t e_{1}-\zeta\right)^{-1}=\sum_{r=1}^{n} A_{r} I_{r}
$$

Taking into account the decompositions (5) and the multiplication table of algebra $\mathbb{A}_{n}^{m}$, we obtain

$$
\begin{gathered}
\sum_{u=1}^{m} I_{u}=1=\left(t e_{1}-\zeta\right)^{-1}\left(t e_{1}-\zeta\right)= \\
=\left(\sum_{u=1}^{m} A_{u} I_{u}+\sum_{r=m+1}^{n} A_{r} I_{r}\right)\left(\sum_{u=1}^{m}\left(t-\xi_{u}\right) I_{u}-\sum_{r=m+1}^{n}\left(y a_{r}+z b_{r}\right) I_{r}\right)= \\
=\sum_{u=1}^{m} A_{u}\left(t-\xi_{u}\right) I_{u}+\left(A_{m+1}\left(t-\xi_{u_{m+1}}\right)+A_{u_{m+1}}\left(-y a_{m+1}-z b_{m+1}\right)\right) I_{m+1}+
\end{gathered}
$$




$$
+\sum_{p=m+2}^{n}\left(A_{u_{p}}\left(-y a_{p}-z b_{p}\right)+A_{p}\left(t-\xi_{u_{p}}\right)+\sum_{r=m+1}^{p-1} A_{r} \sum_{s=m+1}^{p-1}\left(-y a_{s}-z b_{s}\right) \Upsilon_{r, p}^{s}\right) I_{p}
$$

Now, to determine the coefficients $A_{r}$, we have the system of equations:

$$
\begin{aligned}
& A_{u}\left(t-\xi_{u}\right)=1, \quad u=1,2, \ldots, m, A_{m+1}\left(t-\xi_{u_{m+1}}\right)-A_{u_{m+1}} T_{m+1}=0, \\
& -A_{u_{p}} T_{p}+A_{p}\left(t-\xi_{u_{p}}\right)-\sum_{r=m+1}^{p-1} A_{r} B_{r, p}=0, \quad p=m+2, m+3, \ldots, n,
\end{aligned}
$$

where the denotations (10), (11) are used. As immediate consequences of this system, we obtain the equalities (9). The lemma is proved.

It follows from Lemma 1 that the points $(x, y, z) \in \mathbb{R}^{3}$ corresponding to the noninvertible elements $\zeta=x e_{1}+y e_{2}+z e_{3}$ form the straight lines

$$
L_{u}: \quad\left\{\begin{array}{r}
x+y \operatorname{Re} a_{u}+z \operatorname{Re} b_{u}=0, \\
y \operatorname{Im} a_{u}+z \operatorname{Im} b_{u}=0,
\end{array} \quad u=1,2, \ldots, m,\right.
$$

in the three-dimensional space $\mathbb{R}^{3}$.

Lemma 2. If there exists $p \in[m+2, n] \cap \mathbb{N}$ and $r \in[m+1, p-1] \cap \mathbb{N}$ such that $B_{r, p} \neq 0$, then $u_{p}=u_{r}$.

Proof. Since $B_{r, p} \neq 0$, at least one of the numbers $\Upsilon_{m+1, p}^{r}$, $\Upsilon_{m+2, p}^{r}, \ldots, \Upsilon_{p-1, p}^{r}$ is different from zero. Let $\Upsilon_{k, p}^{r} \neq 0$, where $k$ is one of the numbers $m+1, m+2, \ldots, p-1$. The associativity condition of the algebra implies the equality

$$
\left(I_{u_{r}} I_{r}\right) I_{k}=I_{u_{r}}\left(I_{r} I_{k}\right),
$$

which is equivalent to the equality

$$
\sum_{\ell=\max \{k, r\}+1}^{n} \Upsilon_{k, \ell}^{r} I_{\ell}=\sum_{\ell=\max \{k, r\}+1}^{n} \Upsilon_{k, \ell}^{r} I_{u_{r}} I_{\ell}
$$

Note that by the multiplication rule (4), for each $\ell=m+1, m+2, \ldots, n$ the product $I_{u_{r}} I_{\ell}$ is equal to either zero or $I_{\ell}$. Therefore, since $\Upsilon_{k, p}^{r} \neq 0$, the equality (12) implies the equality $\Upsilon_{k, p}^{r} I_{p}=\Upsilon_{k, p}^{r} I_{u_{r}} I_{p}$, i. e. $I_{p}=I_{u_{r}} I_{p}$, and it means that $u_{r}=u_{p}$. The lemma is proved.

Lemma 3. For any $s=m+1, m+2, \ldots, n$ the coefficients $A_{s}$ can be 
expressed in the form

$$
A_{s}=\sum_{k=2}^{s-m+1} \frac{Q_{k, s}}{\left(t-\xi_{u_{s}}\right)^{k}},
$$

where $Q_{k, s}$ are determined by the following recurrence relations:

$$
Q_{2, s}:=T_{s}, \quad Q_{k, s}=\sum_{r=m+1}^{s-1} Q_{k-1, r} B_{r, s}, \quad k=3,4, \ldots, s-m+1 .
$$

Proof. Let us prove the expression (13) by mathematical induction. First, the expression (13) coincides with the equality (9) for $s=m+1$.

Further, suppose the correctness of the formula (13) for all $A_{m+1}$, $A_{m+2}, \ldots, A_{s-1}$ and prove that $A_{s}$ is also expressed by the formula (13). For this purpose, we use the equality (9) for $p=s$. Substituting the expressions (13) for $A_{r}$ in the equality (9), we obtain:

$$
\begin{gathered}
A_{s}=\frac{T_{s}}{\left(t-\xi_{u_{s}}\right)^{2}}+\sum_{r=m+1}^{s-1} \frac{A_{r} B_{r, s}}{t-\xi_{u_{s}}}= \\
=\frac{T_{s}}{\left(t-\xi_{u_{s}}\right)^{2}}+\sum_{r=m+1}^{s-1} \sum_{k=2}^{r-m+1} \frac{Q_{k, r} B_{r, s}}{\left(t-\xi_{u_{s}}\right)\left(t-\xi_{u_{r}}\right)^{k}} .
\end{gathered}
$$

If all $B_{r, s}=0$ for $r=m+1, m+2, \ldots, s-1$, then the formula (15) takes the form (13) with $Q_{2, m+2}=T_{m+2}$ and $Q_{k, s}=0$. Furthermore, for every $r=m+1, m+2, \ldots, s-1$ for which $B_{r, s} \neq 0$, by Lemma $2 u_{r}=u_{s}$, and we

get again the formula (13), where $Q_{k, s}$ are determined by the equalities (14). The lemma is proved.

As a consequence of Lemmas 1, 3, we obtain the following expansion of resolvent:

$$
\left(t e_{1}-\zeta\right)^{-1}=\sum_{u=1}^{m} \frac{1}{t-\xi_{u}} I_{u}+\sum_{s=m+1}^{n} \sum_{k=2}^{s-m+1} \frac{Q_{k, s}}{\left(t-\xi_{u_{s}}\right)^{k}} I_{s} .
$$

\section{A constructive description of monogenic functions.}

Denote $f_{u}\left(E_{3}\right):=\left\{f_{u}(\zeta): \zeta \in E_{3}\right\}$. In what follows, we make the following essential assumption: $f_{u}\left(E_{3}\right)=\mathbb{C}$ for all $u=1,2, \ldots, m$. Obviously, it holds 
if and only if for every fixed $u=1,2, \ldots, m$ at least one of the numbers $a_{u}$ or $b_{u}$ belongs to $\mathbb{C} \backslash \mathbb{R}$.

Lemma 4. Suppose that a domain $\Omega \subset \mathbb{R}^{3}$ is convex in the direction of the straight lines $L_{u}$ and $f_{u}\left(E_{3}\right)=\mathbb{C}$ for all $u=1,2, \ldots, m$. Suppose also that a function $\Phi: \Omega_{\zeta} \rightarrow \mathbb{A}_{n}^{m}$ is monogenic in the domain $\Omega_{\zeta}$. If points $\zeta_{1}, \zeta_{2} \in \Omega_{\zeta}$ such that $\zeta_{2}-\zeta_{1} \in\left\{\zeta=x e_{1}+y e_{2}+z e_{3}:(x, y, z) \in L_{u}\right\}$, then

$$
\Phi\left(\zeta_{1}\right)-\Phi\left(\zeta_{2}\right) \in \mathcal{I}_{u}
$$

The proof of Lemma 4 is similar to the proof of Lemma 1 [8], where one must take the straight line $L_{u}$ and the functional $f_{u}$ instead of the straight line $L$ and the functional $f$, respectively.

Let a domain $\Omega \subset \mathbb{R}^{3}$ be convex in the direction of the straight lines $L_{u}$, $u=1,2, \ldots, m$. By $D_{u}$ we denote that domain in $\mathbb{C}$ onto which the domain $\Omega_{\zeta}$ is mapped by the functional $f_{u}$.

We introduce the linear operators $A_{u}, u=1,2, \ldots, m$, which assign holomorphic functions $F_{u}: D_{u} \rightarrow \mathbb{C}$ to every monogenic function $\Phi: \Omega_{\zeta} \rightarrow \mathbb{A}_{n}^{m}$ by the formula

$$
F_{u}\left(\xi_{u}\right)=f_{u}(\Phi(\zeta))
$$

where $\xi_{u}=f_{u}(\zeta) \equiv x+y a_{u}+z b_{u}$ and $\zeta \in \Omega_{\zeta}$. It follows from Lemma 4 that the value $F_{u}\left(\xi_{u}\right)$ does not depend on a choice of a point $\zeta$ for which $f_{u}(\zeta)=\xi_{u}$.

Lemma 5. Suppose that a domain $\Omega \subset \mathbb{R}^{3}$ is convex in the direction of the straight lines $L_{u}$ and $f_{u}\left(E_{3}\right)=\mathbb{C}$ for all $u=1,2, \ldots, m$. Suppose also that for any fixed $u=1,2, \ldots, m$, a function $F_{u}: D_{u} \rightarrow \mathbb{C}$ is holomorphic in a domain $D_{u}$ and $\Gamma_{u}$ is a closed Jordan rectifiable curve in $D_{u}$ which surrounds the point $\xi_{u}$ and contains no points $\xi_{q}, q=1,2, \ldots, m, q \neq u$. Then the function

$$
\Psi_{u}(\zeta):=I_{u} \int_{\Gamma_{u}} F_{u}(t)\left(t e_{1}-\zeta\right)^{-1} d t
$$

is monogenic in the domain $\Omega_{\zeta}$.

Proof. Let $\zeta \in \Omega_{\zeta}$. First, for any $h \in E_{3}$ and any $\varepsilon>0$, it follows from 
the equality (16) that

$$
\begin{gathered}
I_{u}\left(t e_{1}-\zeta-\varepsilon h\right)^{-1}=\sum_{u=1}^{m} \frac{1}{t-\xi_{u}-\varepsilon f_{u}(h)} I_{u}+ \\
+\sum_{s=m+1}^{n} \sum_{k=2}^{s-m+1} \frac{Q_{k, s}}{\left(t-\xi_{u_{s}}-\varepsilon f_{u_{s}}(h)\right)^{k}} I_{s} I_{u} .
\end{gathered}
$$

Note that for any natural $k$, a complex-value function $1 /\left(t-\xi_{u}-\varepsilon f_{u}(h)\right)^{k}$ tends to the function $1 /\left(t-\xi_{u}\right)^{k}$ uniformly for all $t \in \Gamma_{u}$ when $\varepsilon \rightarrow 0$. Therefore, for any $h \in E_{3}$ the function $I_{u}\left(t e_{1}-\zeta-\varepsilon h\right)^{-1}$ tends to the function $I_{u}\left(t e_{1}-\zeta\right)^{-1}$ uniformly for all $t \in \Gamma_{u}$ when $\varepsilon \rightarrow 0$.

Further, let us prove the existence of Gateaux derivative $\Psi_{u}^{\prime}(\zeta)$ by the definition (6) . Taking into account the Hilbert identity (see, e. g., Theorem 4.8.2 [17, p. 140])

$$
\left(t e_{1}-a\right)^{-1}-\left(t e_{1}-b\right)^{-1}=\left(t e_{1}-a\right)^{-1}\left(t e_{1}-b\right)^{-1}(a-b) \quad \forall a, b \in E_{3},
$$

we have

$$
\begin{aligned}
& \Lambda:=I_{u} \lim _{\varepsilon \rightarrow 0+0} \frac{1}{\varepsilon}\left(\int_{\Gamma_{u}} F_{u}(t)\left(t e_{1}-(\zeta+\varepsilon h)\right)^{-1} d t-\int_{\Gamma_{u}} F_{u}(t)\left(t e_{1}-\zeta\right)^{-1} d t\right)= \\
&=I_{u} h \lim _{\varepsilon \rightarrow 0+0} \int_{\Gamma_{u}} F_{u}(t)\left(t e_{1}-(\zeta+\varepsilon h)\right)^{-1}\left(t e_{1}-\zeta\right)^{-1} d t .
\end{aligned}
$$

Now, since the function $I_{u}\left(t e_{1}-\zeta-\varepsilon h\right)^{-1}$ converges uniformly to the function $I_{u}\left(t e_{1}-\zeta\right)^{-1}$ when $\varepsilon \rightarrow 0$, we obtain the equality

$$
\Lambda=I_{u} h \int_{\Gamma_{u}} F_{u}(t)\left(\left(t e_{1}-\zeta\right)^{-1}\right)^{2} d t
$$

which means the existence of the Gateaux derivative

$$
\Psi_{u}^{\prime}(\zeta):=I_{u} \int_{\Gamma_{u}} F_{u}(t)\left(\left(t e_{1}-\zeta\right)^{-1}\right)^{2} d t .
$$

Finally, in view of the equality (16), components of the expansion of function $\Psi_{u}(\zeta)$ with respect to the basis $\left\{I_{k}\right\}_{k=1}^{n}$ are continuous functions. Therefore, 
the function $\Psi_{u}^{\prime}(\zeta)$ is also continuous, and the function $\Psi_{u}^{\prime}(\zeta)$ is monogenic in $\Omega_{\zeta}$. Lemma is proved.

Lemma 6. Suppose that a domain $\Omega \subset \mathbb{R}^{3}$ is convex in the direction of the straight lines $L_{u}$ and $f_{u}\left(E_{3}\right)=\mathbb{C}$ for all $u=1,2, \ldots, m$. Suppose also that a function $V: \Omega \rightarrow \mathbb{C}$ satisfies the equalities

$$
\frac{\partial V}{\partial y}=\frac{\partial V}{\partial x} a_{u}, \quad \frac{\partial V}{\partial z}=\frac{\partial V}{\partial x} b_{u}
$$

in $\Omega$. Then $V$ is a holomorphic function of the variable $\xi_{u}=f_{u}(\zeta)=x+$ $y a_{u}+z b_{u}$ in the domain $D_{u}$.

Proof. We first separate the real and the imaginary part of the expression

$$
\xi_{u}=x+y \operatorname{Re} a_{u}+z \operatorname{Re} b_{u}+i\left(y \operatorname{Im} a_{u}+z \operatorname{Im} b_{u}\right)=: \tau+i \eta
$$

and note that the equalities (21) yield

$$
\frac{\partial V}{\partial \eta} \operatorname{Im} a_{u}=i \frac{\partial V}{\partial \tau} \operatorname{Im} a_{u}, \quad \frac{\partial V}{\partial \eta} \operatorname{Im} b_{u}=i \frac{\partial V}{\partial \tau} \operatorname{Im} b_{u} .
$$

It follows from the condition $f_{u}\left(E_{3}\right)=\mathbb{C}$ that at least one of the numbers $\operatorname{Im} a_{u}$ or $\operatorname{Im} b_{u}$ is not equal to zero. Therefore, using (23), we get

$$
\frac{\partial V}{\partial \eta}=i \frac{\partial V}{\partial \tau} .
$$

We prove that $V\left(x_{1}, y_{1}, z_{1}\right)=V\left(x_{2}, y_{2}, z_{2}\right)$ for points $\left(x_{1}, y_{1}, z_{1}\right),\left(x_{2}, y_{2}, z_{2}\right) \in$ $\Omega$ such that the segment that connects these points is parallel to the straight line $L_{u}$. To this end, in the domain $\Omega$, we construct two surfaces with common edge, namely a surface $Q$ that contains the point $\left(x_{1}, y_{1}, z_{1}\right)$ and a surface $\Sigma$ that contains the point $\left(x_{2}, y_{2}, z_{2}\right)$, such that the restrictions of the functional $f_{u}$ to the corresponding subsets $Q_{\zeta}:=\left\{\zeta \in E_{3}:(x, y, z) \in Q\right\}$ and $\Sigma_{\zeta}:=\{\zeta \in$ $\left.E_{3}:(x, y, z) \in \Sigma\right\}$ of the domain $\Omega_{\zeta}$ are bijections of these subsets to the same domain $D_{u}$ of the complex plane.

As the surface $Q$ in the domain $\Omega$, we take a fixed equilateral triangle with vertices $A_{u, 1}, A_{u, 2}$, and $A_{u, 3}$ centered at the point $\left(x_{1}, y_{1}, z_{1}\right)$ the plane of which is perpendicular to the straight line $L_{u}$. We now continue the construction of the surface $\Sigma$. 
Consider the triangle with vertices $A_{u, 1}^{\prime}, A_{u, 2}^{\prime}$, and $A_{u, 3}^{\prime}$ centered at the point $\left(x_{2}, y_{2}, z_{2}\right)$, lying in the domain $\Omega$, and such that its sides $A_{u, 1}^{\prime} A_{u, 2}^{\prime}$, $A_{u, 2}^{\prime} A_{u, 3}^{\prime}$, and $A_{u, 1}^{\prime} A_{u, 3}^{\prime}$ are parallel to the segments $A_{u, 1} A_{u, 2}, A_{u, 2} A_{u, 3}$, and $A_{u, 1} A_{u, 3}$, respectively, and have smaller lengths than the sides of the triangle $A_{u, 1} A_{u, 2} A_{u, 3}$. Since the domain $\Omega$ is convex in the direction of the straight line $L_{u}$, we conclude that the prism with vertices $A_{u, 1}^{\prime}, A_{u, 2}^{\prime}, A_{u, 3}^{\prime}, A_{u, 1}^{\prime \prime}, A_{u, 2}^{\prime \prime}$, and $A_{u, 3}^{\prime \prime}$ such that the points $A_{u, 1}^{\prime \prime}, A_{u, 2}^{\prime \prime}$, and $A_{u, 3}^{\prime \prime}$ lie in the plane of the triangle $A_{u, 1} A_{u, 2} A_{u, 3}$ and its edges $A_{u, m}^{\prime} A_{u, m}^{\prime \prime}, m=\overline{1,3}$, are parallel to the straight line $L_{u}$ is completely contained in $\Omega$.

We now fix a triangle with vertices $B_{u, 1}, B_{u, 2}$, and $B_{u, 3}$ such that the point $B_{u, m}$ lies on the segment $A_{u, m}^{\prime} A_{u, m}^{\prime \prime}$ for $m=\overline{1,3}$ and the truncated pyramid with vertices $A_{u, 1}, A_{u, 2}, A_{u, 3}, B_{u, 1}, B_{u, 2}$, and $B_{u, 3}$ and lateral edges $A_{u, m} B_{u, m}, m=\overline{1,3}$, is completely contained in the domain $\Omega$.

Finally, in the plane of the triangle $A_{u, 1}^{\prime} A_{u, 2}^{\prime} A_{u, 3}^{\prime}$, we fix a triangle $T$ with vertices $C_{u, 1}, C_{u, 2}$, and $C_{u, 3}$ such that its sides $C_{u, 1} C_{u, 2}, C_{u, 2} C_{u, 3}$, and $C_{u, 1} C_{u, 3}$ are parallel to the segments $A_{u, 1}^{\prime} A_{u, 2}^{\prime}, A_{u, 2}^{\prime} A_{u, 3}^{\prime}$, and $A_{u, 1}^{\prime} A_{u, 3}^{\prime}$, respectively, and have smaller lengths than the sides of the triangle $A_{u, 1}^{\prime} A_{u, 2}^{\prime} A_{u, 3}^{\prime}$. By construction, the truncated pyramid with vertices $B_{u, 1}, B_{u, 2}, B_{u, 3}, C_{u, 1}, C_{u, 2}$, and $C_{u, 3}$ and lateral edges $B_{u, m} C_{u, m}, m=\overline{1,3}$, is completely contained in the domain $\Omega$.

Let $\Sigma$ denote the surface formed by the triangle $T$ and the lateral surfaces of the truncated pyramids $A_{u, 1} A_{u, 2} A_{u, 3} B_{u, 1} B_{u, 2} B_{u, 3}$ and $B_{u, 1} B_{u, 2} B_{u, 3} C_{u, 1} C_{u, 2} C_{u, 3}$.

Since the surfaces $Q$ and $\Sigma$ have a common edge, the sets $Q_{\zeta}$ and $\Sigma_{\zeta}$ are mapped by the functional $f_{u}$ onto the same domain $D_{u}$ of the complex plane. In the domain $D_{u}$, we introduce two complex-valued functions $H_{u}$ and $W_{u}$ in $D_{u}$ as follows:

$$
\begin{array}{ll}
H_{u}\left(\xi_{u}\right)=V(x, y, z) & \text { for } \quad(x, y, z) \in Q, \\
W_{u}\left(\xi_{u}\right)=V(x, y, z) & \text { for } \quad(x, y, z) \in \Sigma,
\end{array}
$$

where the correspondence between the points $(x, y, z)$ and $\xi_{u} \in D_{u}$ is described by relation (22). 
By virtue of equality (24) and Theorem 6 in [23], the functions $H_{u}$ and $W_{u}$ are holomorphic in the domain $D_{u}$. According to the definition of the functions $H_{u}$ and $W_{u}$, we have $H_{u}\left(\xi_{u}\right) \equiv W_{u}\left(\xi_{u}\right)$ on the boundary of the domain $D_{u}$. By virtue of the holomorphy of the functions $H_{u}$ and $W_{u}$ in the domain $D_{u}$, the identity $H_{u}\left(\xi_{u}\right) \equiv W_{u}\left(\xi_{u}\right)$ holds everywhere in $D_{u}$. Therefore, the equality $V\left(x_{1}, y_{1}, z_{1}\right)=V\left(x_{2}, y_{2}, z_{2}\right)$ is proved.

Thus, a function $V: \Omega \rightarrow \mathbb{C}$ of the form $V(x, y, z):=F\left(\xi_{u}\right)$, where $F\left(\xi_{u}\right)$ is an arbitrary function holomorphic in the domain $D_{u}$, is a general solution of the system (21). The lemma is proved.

Theorem 1. Let a domain $\Omega \subset \mathbb{R}^{3}$ be convex in the direction of the straight lines $L_{u}$ and $f_{u}\left(E_{3}\right)=\mathbb{C}$ for all $u=1,2, \ldots, m$. Then every monogenic function $\Phi: \Omega_{\zeta} \rightarrow \mathbb{A}_{n}^{m}$ can be expressed in the form

$$
\Phi(\zeta)=\sum_{u=1}^{m} I_{u} \frac{1}{2 \pi i} \int_{\Gamma_{u}} F_{u}(t)\left(t e_{1}-\zeta\right)^{-1} d t+\sum_{s=m+1}^{n} I_{s} \frac{1}{2 \pi i} \int_{\Gamma_{u_{s}}} G_{s}(t)\left(t e_{1}-\zeta\right)^{-1} d t
$$

where $F_{u}$ and $G_{s}$ are certain holomorphic functions in the domains $D_{u}$ and $D_{u_{s}}$, respectively, and $\Gamma_{q}$ is a closed Jordan rectifiable curve in $D_{q}$ which surrounds the point $\xi_{q}$ and contains no points $\xi_{\ell}, \ell, q=1,2, \ldots, m, \ell \neq q$.

Proof. We set

$$
F_{u}:=A_{u} \Phi, \quad u=1,2, \ldots, m .
$$

Let us show that the values of monogenic function

$$
\Phi_{0}(\zeta):=\Phi(\zeta)-\sum_{u=1}^{m} I_{u} \frac{1}{2 \pi i} \int_{\Gamma_{u}} F_{u}(t)\left(t e_{1}-\zeta\right)^{-1} d t
$$

belong to the radical $\mathcal{R}$, i. e. $\Phi_{0}(\zeta) \in \mathcal{R}$ for all $\zeta \in \Omega_{\zeta}$. As a consequence of the equality (16), we have the equality

$$
\begin{gathered}
I_{u} \frac{1}{2 \pi i} \int_{\Gamma_{u}} F_{u}(t)\left(t e_{1}-\zeta\right)^{-1} d t=I_{u} \frac{1}{2 \pi i} \int_{\Gamma_{u}} \frac{F_{u}(t)}{t-\xi_{u}} d t+ \\
+\frac{1}{2 \pi i} \sum_{s=m+1}^{n} \sum_{k=2}^{s-m+1} \int_{\Gamma_{u}} \frac{F_{u}(t) Q_{k, s}}{\left(t-\xi_{u_{s}}\right)^{k}} d t I_{s} I_{u}
\end{gathered}
$$


from which we obtain the equality

$$
f_{u}\left(\sum_{u=1}^{m} I_{u} \frac{1}{2 \pi i} \int_{\Gamma_{u}} F_{u}(t)\left(t e_{1}-\zeta\right)^{-1} d t\right)=F_{u}\left(\xi_{u}\right) .
$$

Operating onto the equality (27) by the functional $f_{u}$ and taking into account the relations (18), (26) $)$ (28), we get the equality

$$
f_{u}\left(\Phi_{0}(\zeta)\right)=F_{u}\left(\xi_{u}\right)-F_{u}\left(\xi_{u}\right)=0
$$

for all $u=1,2, \ldots, m$, i. e. $\Phi_{0}(\zeta) \in \mathcal{R}$.

Therefore, the function $\Phi_{0}$ is of the form

$$
\Phi_{0}(\zeta)=\sum_{s=m+1}^{n} V_{s}(x, y, z) I_{s},
$$

where $V_{s}: \Omega \rightarrow \mathbb{C}$, and the Cauchy - Riemann conditions (8) are satisfied with $\Phi=\Phi_{0}$. Substituting the expressions (5), (29) into the equality (8), we obtain

$$
\begin{gathered}
\sum_{s=m+1}^{n} \frac{\partial V_{s}}{\partial y} I_{s}=\sum_{s=m+1}^{n} \frac{\partial V_{s}}{\partial x} I_{s} \sum_{r=1}^{n} a_{r} I_{r}, \\
\sum_{s=m+1}^{n} \frac{\partial V_{s}}{\partial z} I_{s}=\sum_{s=m+1}^{n} \frac{\partial V_{s}}{\partial x} I_{s} \sum_{r=1}^{n} b_{r} I_{r} .
\end{gathered}
$$

Equating the coefficients of $I_{m+1}$ in these equalities, we obtain the following system of equations for determining the function $V_{m+1}(x, y, z)$ :

$$
\frac{\partial V_{m+1}}{\partial y}=\frac{\partial V_{m+1}}{\partial x} a_{u_{m+1}}, \quad \frac{\partial V_{m+1}}{\partial z}=\frac{\partial V_{m+1}}{\partial x} b_{u_{m+1}} .
$$

It follows from Lemma 6 that $V_{m+1}(x, y, z) \equiv G_{m+1}\left(\xi_{u_{m+1}}\right)$, where $G_{m+1}$ is a function holomorphic in the domain $D_{u_{m+1}}$. Therefore,

$$
\Phi_{0}(\zeta)=G_{m+1}\left(\xi_{u_{m+1}}\right) I_{m+1}+\sum_{s=m+2}^{n} V_{s}(x, y, z) I_{s} .
$$

Due to the expansion (16), we have the representation

$$
I_{m+1} \frac{1}{2 \pi i} \int_{\Gamma_{u_{m+1}}} G_{m+1}(t)\left(t e_{1}-\zeta\right)^{-1} d t=G_{m+1}\left(\xi_{u_{m+1}}\right) I_{m+1}+\Psi(\zeta),
$$

where $\Psi(\zeta)$ is a function with values in the set $\left\{\sum_{k=m+2}^{n} \alpha_{k} I_{k}: \alpha_{k} \in \mathbb{C}\right\}$. 
Now, consider the function

$$
\Phi_{1}(\zeta):=\Phi_{0}(\zeta)-I_{m+1} \frac{1}{2 \pi i} \int_{\Gamma_{u_{m+1}}} G_{m+1}(t)\left(t e_{1}-\zeta\right)^{-1} d t .
$$

In view of the relations (31), (32), $\Phi_{1}$ can be represented in the form

$$
\Phi_{1}(\zeta)=\sum_{s=m+2}^{n} \widetilde{V}_{s}(x, y, z) I_{s}
$$

where $\widetilde{V}_{s}: \Omega \rightarrow \mathbb{C}$.

Inasmuch as $\Phi_{1}$ is a monogenic function in $\Omega_{\zeta}$, the functions $\widetilde{V}_{m+2}, \widetilde{V}_{m+3}, \ldots, \widetilde{V}_{n}$ satisfy the system (30), where $V_{m+1} \equiv 0, V_{s}=\widetilde{V}_{s}$ for $s=m+2, m+3, \ldots, n$. Therefore, similarly to the function $V_{m+1}(x, y, z) \equiv$ $G_{m+1}\left(\xi_{u_{m+1}}\right)$, the function $\widetilde{V}_{m+2}$ satisfies the equations

$$
\frac{\partial \widetilde{V}_{m+2}}{\partial y}=\frac{\partial \widetilde{V}_{m+2}}{\partial x} a_{u_{m+2}}, \quad \frac{\partial \widetilde{V}_{m+2}}{\partial z}=\frac{\partial \widetilde{V}_{m+2}}{\partial x} b_{u_{m+2}}
$$

and is of the form $\widetilde{V}_{m+2}(x, y, z) \equiv G_{m+2}\left(\xi_{u_{m+2}}\right)$, where $G_{m+2}$ is a function holomorphic in the domain $D_{u_{m+2}}$.

In such a way, step by step, considering the functions

$$
\Phi_{j}(\zeta):=\Phi_{j-1}(\zeta)-I_{m+j} \frac{1}{2 \pi i} \int_{\Gamma_{u_{m+j}}} G_{m+j}(t)\left(t e_{1}-\zeta\right)^{-1} d t
$$

for $j=2,3, \ldots, n-m-1$, we get the representation (25) of the function $\Phi$. The theorem is proved.

Taking into account the expansion (16), one can rewrite the equality (25) in the following equivalent form:

$$
\begin{gathered}
\Phi(\zeta)=\sum_{u=1}^{m} F_{u}\left(\xi_{u}\right) I_{u}+\sum_{s=m+1}^{n} \sum_{k=2}^{s-m+1} \frac{1}{(k-1) !} Q_{k, s} F_{u_{s}}^{(k-1)}\left(\xi_{u_{s}}\right) I_{s}+ \\
+\sum_{q=m+1}^{n} G_{q}\left(\xi_{u_{q}}\right) I_{q}+\sum_{q=m+1}^{n} \sum_{s=m+1}^{n} \sum_{k=2}^{s-m+1} \frac{1}{(k-1) !} Q_{k, s} G_{q}^{(k-1)}\left(\xi_{u_{q}}\right) I_{q} I_{s} .
\end{gathered}
$$

Thus, the equalities (25) and (33) specify methods to construct explicitly any monogenic functions $\Phi: \Omega_{\zeta} \rightarrow \mathbb{A}_{n}^{m}$ using $n$ corresponding holomorphic functions of complex variables. 
The following statement follows immediately from the equality (33) in which the right-hand side is a monogenic function in the domain $\Pi_{\zeta}:=\left\{\zeta \in E_{3}\right.$ : $\left.f_{u}(\zeta)=D_{u}, u=1,2, \ldots, m\right\}$.

Theorem 2. Let a domain $\Omega$ be convex in the directions of the straight lines $L_{u}$ and $f_{u}\left(E_{3}\right)=\mathbb{C}$ for all $u=1,2, \ldots, m$. Then every monogenic function $\Phi: \Omega_{\zeta} \rightarrow \mathbb{A}_{n}^{m}$ can be continued to a function monogenic in the domain $\Pi_{\zeta}$.

The next statement is a fundamental consequence of the equality (33), and it is true for an arbitrary domain $\Omega_{\zeta}$.

Theorem 3. Let $f_{u}\left(E_{3}\right)=\mathbb{C}$ for all $u=1,2, \ldots, m$. Then for every monogenic function $\Phi: \Omega_{\zeta} \rightarrow \mathbb{A}_{n}^{m}$ in an arbitrary domain $\Omega_{\zeta}$, the Gateaux $r$-th derivatives $\Phi^{(r)}$ are monogenic functions in $\Omega_{\zeta}$ for all $r$.

The proof is completely analogous to the proof of Theorem 4 [8].

Using the integral expression (25) of monogenic function $\Phi: \Omega_{\zeta} \rightarrow \mathbb{A}_{n}^{m}$ in the case where a domain $\Omega$ is convex in the directions of the straight lines $L_{u}, u=1,2, \ldots, m$, we obtain the following expression for the Gateaux $r$-th derivative $\Phi^{(r)}$ :

$$
\begin{gathered}
\Phi^{(r)}(\zeta)=\sum_{u=1}^{m} I_{u} \frac{r !}{2 \pi i} \int_{\Gamma_{u}} F_{u}(t)\left((t-\zeta)^{-1}\right)^{r+1} d t+ \\
+\sum_{s=m+1}^{n} I_{s} \frac{r !}{2 \pi i} \int_{\Gamma_{u_{s}}} G_{s}(t)\left((t-\zeta)^{-1}\right)^{r+1} d t \quad \forall \zeta \in \Omega_{\zeta} .
\end{gathered}
$$

\section{Special cases.}

Note that in the cases where the algebra $\mathbb{A}_{n}^{m}$ has some specific properties (for instance, properties described in Propositions 1 and 2), it is easy to simplify the form of the equality (33).

1. In the case considered in Proposition 1, the following equalities hold:

$$
u_{m+1}=u_{m+2}=\ldots=u_{n}=: \eta
$$


In this case the representation (33) takes the form

$$
\begin{gathered}
\Phi(\zeta)=\sum_{u=1}^{m} F_{u}\left(\xi_{u}\right) I_{u}+\sum_{s=m+1}^{n} \sum_{k=2}^{s-m+1} \frac{1}{(k-1) !} Q_{k, s} F_{\eta}^{(k-1)}\left(\xi_{\eta}\right) I_{s}+ \\
+\sum_{s=m+1}^{n} G_{s}\left(\xi_{\eta}\right) I_{s}+\sum_{q=m+1}^{n} \sum_{s=m+1}^{n} \sum_{k=2}^{s-m+1} \frac{1}{(k-1) !} Q_{k, s} G_{q}^{(k-1)}\left(\xi_{\eta}\right) I_{s} I_{q} .
\end{gathered}
$$

The formula (34) generalizes representations of monogenic functions in both three-dimensional harmonic algebras (see [8, 9, 10]) and specific $n$-dimensional algebras (see [13, 14]) to the case of algebras more general form.

2. In the case considered in Proposition 2, the functions $B_{r, p}$ from the equalities (11) are identically equal to zero. In this case the representation (16) takes the form

$$
\left(t e_{1}-\zeta\right)^{-1}=\sum_{u=1}^{m} \frac{1}{t-\xi_{u}} I_{u}+\sum_{s=m+1}^{n} \frac{T_{s}}{\left(t-\xi_{u_{s}}\right)^{2}} I_{s},
$$

and as a consequence of the equalities (25), (35), we obtain the following representation of monogenic function:

$$
\Phi(\zeta)=\sum_{u=1}^{m} F_{u}\left(\xi_{u}\right) I_{u}+\sum_{s=m+1}^{n} G_{s}\left(\xi_{u_{s}}\right) I_{s}+\sum_{s=m+1}^{n} T_{s} F_{u_{s}}^{\prime}\left(\xi_{u_{s}}\right) I_{s} .
$$

The formula (36) generalizes representations of monogenic functions in both a three-dimensional harmonic algebra with one-dimensional radical (see [9]) and semi-simple algebras (see [10, 14]) to the case of algebras more general form.

3. In the case where $n=m$, the algebra $\mathbb{A}_{n}^{n}$ is semi-simple and contains no nilpotent subalgebra. Then the formulas (34), (36) take the form

$$
\Phi(\zeta)=\sum_{u=1}^{n} F_{u}\left(\xi_{u}\right) I_{u}
$$

because there are no vectors $\left\{I_{k}\right\}_{k=m+1}^{n}$. This formula was obtained in the paper [14]. 


\section{The relations between monogenic functions and partial differential equations.}

Consider the following linear partial differential equation with constant coefficients:

$$
\mathcal{L}_{N} U(x, y, z):=\sum_{\alpha+\beta+\gamma=N} C_{\alpha, \beta, \gamma} \frac{\partial^{N} U}{\partial x^{\alpha} \partial y^{\beta} \partial z^{\gamma}}=0, \quad C_{\alpha, \beta, \gamma} \in \mathbb{R} .
$$

If a function $\Phi(\zeta)$ is $N$-times differentiable in the sense of Gateaux in every point of $\Omega_{\zeta}$, then

$$
\frac{\partial^{\alpha+\beta+\gamma} \Phi}{\partial x^{\alpha} \partial y^{\beta} \partial z^{\gamma}}=e_{1}^{\alpha} e_{2}^{\beta} e_{3}^{\gamma} \Phi^{(\alpha+\beta+\gamma)}(\zeta)=e_{2}^{\beta} e_{3}^{\gamma} \Phi^{(N)}(\zeta) .
$$

Therefore, due to the equality

$$
\mathcal{L}_{N} \Phi(\zeta)=\Phi^{(N)}(\zeta) \sum_{\alpha+\beta+\gamma=N} C_{\alpha, \beta, \gamma} e_{2}^{\beta} e_{3}^{\gamma},
$$

every $N$-times differentiable in the sense of Gateaux in $\Omega_{\zeta}$ function $\Phi$ satisfies the equation $\mathcal{L}_{N} \Phi(\zeta)=0$ everywhere in $\Omega_{\zeta}$ if and only if

$$
\sum_{\alpha+\beta+\gamma=N} C_{\alpha, \beta, \gamma} e_{2}^{\beta} e_{3}^{\gamma}=0 .
$$

Accordingly, if the condition (39) is satisfied, then the real-valued components $\operatorname{Re} U_{k}(x, y, z)$ and $\operatorname{Im} U_{k}(x, y, z)$ of the decomposition (7) are solutions of the equation (37).

In the case where $f_{u}\left(E_{3}\right)=\mathbb{C}$ for all $u=1,2, \ldots, m$, it follows from Theorem 3 that the equality (38) holds for every monogenic function $\Phi: \Omega_{\zeta} \rightarrow \mathbb{A}_{n}^{m}$.

Thus, to construct solutions of the equation (37) in the form of components of monogenic functions, we must to find a triad of linearly independent over the field $\mathbb{R}$ vectors (5) satisfying the characteristic equation (39) and to verify the condition: $f_{u}\left(E_{3}\right)=\mathbb{C}$ for all $u=1,2, \ldots, m$. Then, the formula (25) gives a constructive description of all mentioned monogenic functions.

In the next theorem, we assign a special class of equations (37) for which $f_{u}\left(E_{3}\right)=\mathbb{C}$ for all $u=1,2, \ldots, m$. Let us introduce the polynomial

$$
P(a, b):=\sum_{\alpha+\beta+\gamma=N} C_{\alpha, \beta, \gamma} a^{\beta} b^{\gamma} .
$$


Theorem 4. Suppose that there exist linearly independent over the field $\mathbb{R}$ vectors $e_{1}, e_{2}, e_{3}$ in $\mathbb{A}_{n}^{m}$ of the form (5) that satisfy the equality (39). If $P(a, b) \neq 0$ for all real $a$ and $b$, then $f_{u}\left(E_{3}\right)=\mathbb{C}$ for all $u=1,2, \ldots, m$.

Proof. Using the multiplication table of $\mathbb{A}_{n}^{m}$, we obtain the equalities

$$
e_{2}^{\beta}=\sum_{u=1}^{m} a_{u}^{\beta} I_{u}+\Psi_{\mathcal{R}}, \quad e_{3}^{\gamma}=\sum_{u=1}^{m} b_{u}^{\gamma} I_{u}+\Theta_{\mathcal{R}},
$$

where $\Psi_{\mathcal{R}}, \Theta_{\mathcal{R}} \in \mathcal{R}$. Now the equality (39) takes the form

$$
\sum_{\alpha+\beta+\gamma=N} C_{\alpha, \beta, \gamma}\left(\sum_{u=1}^{m} a_{u}^{\beta} b_{u}^{\gamma} I_{u}+\widetilde{\Psi}_{\mathcal{R}}\right)=0,
$$

where $\widetilde{\Psi}_{\mathcal{R}} \in \mathcal{R}$. Moreover, due to the assumption that the vectors $e_{1}, e_{2}, e_{3}$ of the form (5) satisfy the equality (39), there exist complex coefficients $a_{k}, b_{k}$ for $k=1,2, \ldots, n$ that satisfy the equality (41).

It follows from the equality (41) that

$$
\sum_{\alpha+\beta+\gamma=n} C_{\alpha, \beta, \gamma} a_{u}^{\beta} b_{u}^{\gamma}=0, \quad u=1,2, \ldots, m .
$$

Since $P(a, b) \neq 0$ for all $a, b \in \mathbb{R}$, the equalities (42) can be satisfied only if for each $u=1,2, \ldots, m$ at least one of the numbers $a_{u}$ or $b_{u}$ belongs to $\mathbb{C} \backslash \mathbb{R}$ that implies the relation $f_{u}\left(E_{3}\right)=\mathbb{C}$ for all $u=1,2, \ldots, m$. The theorem is proved.

Note that if $P(a, b) \neq 0$ for all $a, b \in \mathbb{R}$, then $C_{N, 0,0} \neq 0$ because otherwise $P(a, b)=0$ for $a=b=0$.

Since the function $P(a, b)$ is continuous on $\mathbb{R}^{2}$, the condition $P(a, b) \neq 0$ means either $P(a, b)>0$ or $P(a, b)<0$ for all $a, b \in \mathbb{R}$. Therefore, it is obvious that for any equation (37) of elliptic type, the condition $P(a, b) \neq 0$ is always satisfied for all $a, b \in \mathbb{R}$. At the same time, there are equations (37) for which $P(a, b)>0$ for all $a, b \in \mathbb{R}$, but which are not elliptic. For example, such are the equations

$$
\frac{\partial^{3} u}{\partial x^{3}}+\frac{\partial^{3} u}{\partial x \partial y^{2}}+\frac{\partial^{3} u}{\partial x \partial z^{2}}=0 \text { and } \frac{\partial^{5} u}{\partial x^{5}}+\frac{\partial^{5} u}{\partial x^{3} \partial y^{2}}+\frac{\partial^{5} u}{\partial x \partial y^{2} \partial z^{2}}=0
$$

considered in $\mathbb{R}^{3}$, in particular. 
Acknowledgements. The author expresses a gratitude to Professor S. A. Plaksa and Mr. R. P. Pukhtaievych for numerous discussions and valuable advices.

\section{References}

[1] C. Segre, The real representations of complex elements and extentions to bicomlex systems, Math. Ann., 40 (1892), 413-467.

[2] F. Ringleb, Beiträge zur funktionentheorie in hyperkomplexen systemen, I, Rend. Circ. Mat. Palermo, 57 (1933), no. 1, 311-340.

[3] J. D. Riley, Contributions to the theory of functions of a bicomplex variable, Tohoku Math. J., 5 (1953), no. 2, 132-165.

[4] P. W. Ketchum, Analytic functions of hypercomplex variables, Trans. Amer. Math. Soc., 30 (1928), no. 4, 641-667.

[5] I. P. Mel'nichenko, The representation of harmonic mappings by monogenic functions, Ukr. Math. J., 27 (1975), no. 5, 499-505.

[6] I. P. Mel'nichenko, Algebras of functionally invariant solutions of the threedimensional Laplace equation, Ukr. Math. J., 55 (2003), no. 9, 1551-1557.

[7] I. P. Mel'nichenko and S. A. Plaksa, Commutative algebras and spatial potential fields, Kiev, Inst. Math. NAS Ukraine, 2008 [in Russian].

[8] S. A. Plaksa and V. S. Shpakovskii, Constructive description of monogenic functions in a harmonic algebra of the third rank, Ukr. Math. J., 62 (2011), no. 8, 1251-1266.

[9] S. A. Plaksa and R. P. Pukhtaevich, Constructive description of monogenic functions in a three-dimensional harmonic algebra with one-dimensional radical, Ukr. Math. J., 65 (2013), no. 5, 740-751. 
[10] R. P. Pukhtaievych, Monogenic functions in a three-dimensional harmonic semi-simple algebra, Zb. Pr. Inst. Mat. NAN Ukr., 10 (2013), no. 4-5, $352-361$.

[11] V. S. Shpakivskyi and S. A. Plaksa, Integral theorems and a Cauchy formula in a commutative three-dimensional harmonic algebra, Bulletin Soc. Sci. Lettr. Lódź, 60 (2010), 47-54.

[12] S. A. Plaksa, Commutative algebras associated with classic equations of mathematical physics, Advances in Applied Analysis, Trends in Mathematics, Springer, Basel, 2012, 177-223.

[13] S. A. Plaksa and V. S. Shpakivskyi, Monogenic functions in a finitedimensional algebra with unit and radical of maximal dimensionality, J. Algerian Math. Soc., 1 (2014), 1-13.

[14] S. A. Plaksa and R. P. Pukhtaievych, Constructive description of monogenic functions in $n$-dimensional semi-simple algebra, An. Şt. Univ. Ovidius Constanţa, 22(2014), no. 1, 221-235.

[15] E. Cartan, Les groupes bilinéares et les systèmes de nombres complexes, Annales de la faculté des sciences de Toulouse, 12(1898), no. 1, 1-64.

[16] D. Burde and W. de Graaf, Classification of Novicov algebras, Applicable Algebra in Engineering, Communication and Computing, 24(2013), no. 1, $1-15$.

[17] E. Hille and R. S. Phillips, Functional analysis and semi-groups [Russian translation], Inostr. Lit., Moscow (1962).

[18] F. Brackx and R. Delanghe, Duality in hypercomplex functions theory, J. Funct. Anal., 37(1980), no. 2, 164-181.

[19] J. Ryan, Dirac operators, conformal transformations and aspects of classical harmonic analysis, J. Lie Theory, 8(1998), 67-82.

[20] A. Sudbery, Quaternionic analysis, Math. Proc. Camb. Phil. Soc., 85(1979), 199-225. 
[21] V. V. Kravchenko and M. V. Shapiro, Integral representations for spetial models of mathematical physics, Pitman Research Notes in Mathematics, Addison Wesley Longman Inc. (1996).

[22] W. Sprössig, Quaternionic analysis and Maxwell's equations, CUBO A Math. J., 7(2005), no. 2, 57-67.

[23] G. P. Tolstov, On curvilinear and iterated integrals, Tr. Mat. Inst. Akad. Nauk SSSR, 35(1950), 3-101 [in Russian].

Vitalii Shpakivskyi

Department of Complex Analysis and Potential Theory

Institute of Mathematics of the National Academy of Sciences of Ukraine, 3, Tereshchenkivs'ka st.

01601 Kyiv-4

UKRAINE

http://www.imath.kiev.ua/ complex/

e-mail: shpakivskyi@mail.ru,shpakivskyi@imath.kiev.ua 